Res., Soc. Dev. 2019; 8(10):e318101384

ISSN 2525-3409 | DOI: http://dx.doi.org/10.33448/rsd-v8i10.1384

\title{
Evolução da construção de um modelo pedagógico para atividades de m-learning
}

Evolution of the construction of a pedagogical model for m-learning activities

Evolución de la construcción de un modelo pedagógico para actividades de m-learning

Recebido: 05/07/2019 | Revisado: 10/08/2019 | Aceito: 13/08/2019 | Publicado: 23/08/2019

\section{Ernane Rosa Martins}

ORCID: https://orcid.org/0000-0002-1543-1108

Instituto Federal de Educação Ciência e Tecnologia de Goiás, Brasil

E-mail: ernane.martins@ifg.edu.br

Luís Borges Gouveia

ORCID: https://orcid.org/0000-0002-2079-3234

Universidade Fernando Pessoa, Portugal

E-mail: lmbg@ufp.edu.pt

\section{Resumo}

O objetivo deste artigo é apresentar à evolução da construção de um modelo pedagógico para m-learning, denominado de ML-SAI, que foi fundamentado no conceito de Sala de Aula Invertida (SAI). Nesse sentido, inicialmente, é definido o que este estudo entende por modelo pedagógico, m-learning e Sala de Aula Invertida. Em seguida são apresentados as metodologias e os procedimentos para a elaboração do mesmo. Em seguida, é detalhado o modelo pedagógico ML-SAI, descrevendo a sua arquitetura e fornecendo algumas sugestões de estratégias para a orientação de professores interessados em utiliza-lo no desenvolvimento de atividades de m-learning. Por fim, é relatado uma experimentação do modelo pedagógico proposto, realizada por meio de uma atividade prática em um curso de Sistemas de Informação. A proposição do ML-SAI apresentou bons resultados, permitindo colaborar no planejamento e desenvolvimento consistente das práticas pedagógicas e melhor aproveitamento dos recursos tecnológicos, fornecendo algumas sugestões de estratégias a professores e pesquisadores interessados em utiliza-lo, orientando estes no desenvolvimento das atividades de m-learning. Sendo bem aceito no teste realizado.

Palavras-chave: Modelo pedagógico; Sala de aula invertida; Mobile learning; Aprendizagem.

\section{Abstract}


The purpose of this article is to present the evolution of the construction of a pedagogical model for $\mathrm{m}$ learning, called ML-SAI, which was based on the concept of Inverted Classroom. In this sense, it is initially defined what this study understands by pedagogical model, m-learning and Inverted Classroom. Following are the methodologies and procedures for the elaboration of the same. Next, the ML-SAI pedagogical model is described, describing its architecture and providing some suggestions of strategies for the orientation of teachers interested in using it in the development of m-learning activities. Finally, it is reported an experimentation of the proposed pedagogical model, accomplished through a practical activity in an Information Systems course. The ML-SAI proposal presented good results, allowing collaboration in the planning and consistent development of pedagogical practices and better utilization of technological resources, providing some suggestions of strategies to teachers and researchers interested in using it, guiding them in the development of activities of $m$-learning. Being well accepted in the test performed.

Keywords: Pedagogical model; Reverse classroom; Mobile learning; Learning.

\section{Resumen}

El propósito de este artículo es presentar la evolución de la construcción de un modelo pedagógico para m-learning, llamado ML-SAI, que se basó en el concepto de Aula Invertida. En este sentido, inicialmente se define lo que este estudio entiende por modelo pedagógico, m-learning y Aula invertida. A continuación se detallan las metodologías y procedimientos para la elaboración de los mismos. A continuación, se describe el modelo pedagógico de ML-SAI, que describe su arquitectura y proporciona algunas sugerencias de estrategias para la orientación de los maestros interesados en utilizarla en el desarrollo de actividades de m-learning. Finalmente, se reporta una experimentación del modelo pedagógico propuesto, realizado a través de una actividad práctica en un curso de Sistemas de Información. La propuesta de ML-SAI presentó buenos resultados, permitiendo la colaboración en la planificación y el desarrollo consistente de prácticas pedagógicas y una mejor utilización de los recursos tecnológicos, brindando algunas sugerencias de estrategias a docentes e investigadores interesados en usarlas, guiándolas en el desarrollo de actividades de $\mathrm{m}$-aprendizaje Siendo bien aceptado en la prueba realizada.

Palabras clave: Modelo pedagógico; Aula inversa; Aprendizaje móvil; Aprendizaje.

\section{Introdução}

O uso das Tecnologias de Informação e Comunicação (TIC) em sala de aula podem trazer melhorias significativas para a aprendizagem, pois, segundo Moran (2013) "com as tecnologias atuais, a escola pode transformar-se em um conjunto de espaços ricos de aprendizagens significativas, presenciais e digitais, que motivem os alunos a aprender 
ativamente, a pesquisar o tempo todo, a serem proativos, a saber tomar iniciativas, interagir". Assim, é fundamental que o professor contemporâneo busque trazer as novas tecnologias digitais para a sua vivência e prática pedagógica escolar, visto que os alunos já estão utilizando e adaptados a estas novas tendências tecnológicas.

A utilização de ferramentas digitais, quando feita de maneira planejada, pode contribuir no desenvolvimento das habilidades e competências dos alunos de forma autônoma a partir das suas necessidades, facilitando e trazendo mais inovação e dinamicidade aos estudos. A escola e os professores necessitam atualizar as suas práticas pedagógicas para promover a utilização das novas ferramentas digitais nas atividades acadêmicas, de modo a acompanhar a evolução dos alunos. Assim, o objetivo deste trabalho é apresenta a evolução na construção do modelo pedagógico para m-learning, denominado de ML-SAI, que foi fundamentado no conceito de Sala de Aula Invertida e sua experimentação.

\section{Conceituação teórica}

Para a realização deste trabalho foi preciso estabelecer algumas definições orientadoras da pesquisa. Assim, a definição de Sala de Aula Invertida utilizada neste trabalho é "o que antes era feita na sala de aula no modelo tradicional, agora é executado em casa, enquanto que as atividades que eram realizadas sozinhas pelos alunos como tarefa de casa, agora são executadas em sala de aula” (Bergmann \& Sams, 2016).

Adotou-se também a definição para m-learning de Wains \& Mahmood (2008) como sendo "um campo emergente, que engloba tecnologias sem fio e computação móvel para permitir que a aprendizagem possa ocorrer em qualquer tempo e em qualquer lugar, maximizando a liberdade dos alunos".

O m-learning permite aos alunos ter uma utilização mais flexível e otimizada do tempo, pois podem usar as tecnologias sob demanda, em qualquer lugar, em qualquer momento, ampliando a possibilidade de uso educacional de recursos que já estão sendo utilizados para comunicação e lazer. Os dispositivos móveis possuem muito potencial, permitindo oferecer diferentes níveis de envolvimento, melhorando o desempenho dos estudantes, pela colaboração ou mesmo pelos diversos formatos de materiais disponíveis, como vídeos, imagens, áudios e texto (Barcelos, Tarouco \& Berch, 2009).

Outra definição adotada foi a de modelo pedagógico de Behar, Passerino \& Bernardi (2007) que afirma ser: “um sistema de premissas teórica que representa, explica e orienta a 
forma como se aborda o currículo e que se concretiza nas práticas pedagógicas e nas interações professor-aluno-objeto de conhecimento".

Segundo Behar (2009) o modelo pedagógico deve estar baseado em duas ou mais teorias educacionais utilizadas como eixo norteador da aprendizagem e nas experiências pessoais, criando o que ela denomina de Modelo Pessoal. Conforme a mesma autora este é fundamentado em dois elementos: Arquitetura Pedagógica (AP) e Estratégias para a Aplicação da mesma. A AP é a estrutura principal do modelo e as estratégias para a aplicação da AP são as dinâmicas do modelo pedagógico, que dependem do contexto e variáveis que envolvem o processo educativo. Estas ações didáticas direcionam o professor para colocar em pratica a AP a um contexto particular, tornando-a individual.

As tecnologias por si não são transformadoras dos contextos educacionais. Simplesmente inseri-las na educação, sem criar uma proposta didático-pedagógica consistente, coerente e com um planejamento alinhado com as necessidades dos alunos, não permite explorar suas potencialidades e possibilitar o desenvolvimento de práticas educacionais diferenciadas. São várias as possibilidades, mas as tecnologias apenas serão bem utilizadas no contexto educacional se as propostas metodológicas forem suficientemente abertas, criativas e focadas no aprendiz (Costa, et al., 2014).

\section{Metodologia}

Este presente estudo tem como principal abordagem metodológica a pesquisa exploratória. De acordo com Gil (1999) a pesquisa exploratória visa desenvolver, esclarecer ou modificar conceitos e ideias sobre determinado assunto.

O método utilizado nesta pesquisa foi o estudo de caso, este foi escolhido por ser um estudo de natureza empírica que investiga um determinado fenômeno, geralmente contemporâneo, dentro de um contexto real de vida, quando as fronteiras entre o fenômeno e o contexto em que se insere não são claramente definidas, tratando-se de uma análise aprofundada de um ou mais objetos, permitindo seu amplo e detalhado conhecimento (Gil, 2002).

Após a elaboração do modelo pedagógico ML-SAI, foi realizada uma atividade orientada pelo modelo, com alunos da disciplina de programação do curso superior de Sistemas de Informação (SI), que teve como objetivo a compreensão de alguns algoritmos selecionados pelo professor. 
Para coleta de dados, foram realizadas três perguntas por meio de questionário impresso a vinte e seis alunos da disciplina. A recolha de dados possibilitou sustentar a parte empírica da investigação. Foram realizadas duas perguntas fechadas e uma aberta. As respostas fechadas consideraram uma escala de avaliação composta por: 5 - concordo plenamente, 4 - concordo, 3 - não concordo nem discordo, 2 - discordo, 1 - discordo completamente. Convém realçar que a opção por uma escala de cinco níveis justificou-se por querer obter uma percepção mais precisa das respostas dos participantes.

\section{Elaboração do modelo pedagógico}

O modelo pedagógico proposto foi elaboração a partir da análise do referencial bibliográfico levantado, dos resultados de pesquisas exploratórias realizadas utilizando o conceito de Sala de Aula Invertida combinada com alguma tecnologia móvel, como Kahoot, WhatsApp e Facebook e das experimentações com o próprio modelo pedagógico.

Este foi desenvolvido considerando: aspectos organizacionais, metodológicos, tecnológicos e relativos ao conteúdo e sendo propostas estratégias relacionadas a estes. Sendo ainda submetido à análise de três especialistas com experiências na área pedagógica, com anos de atuação com m-learning. Tendo como finalidade principal a construção de um modelo pedagógico inédito para atividades de m-learning, que seja credível e válido do ponto de vista científico, pedagógico e por seus destinatários, especificou-se três etapas de elaboração do mesmo, sendo elas:

Fase 1: Busca por modelos pedagógicos com características similares ao proposto e realização de uma exaustiva revisão da literatura, para concepção da versão preliminar do modelo pedagógico, denominado de modelo pedagógico prévio.

Fase 2: Realização de estudos preliminares formados por pesquisa exploratória com estudos de casos, que orientaram uma reformulação e melhoramento do modelo pedagógico prévio, gerando um modelo pedagógico intermediário,

Fase 3: Experimentação do modelo pedagógico intermediário, em conjunto com análise e sugestões de especialistas de relevo na área pedagógica com anos de atuação na área, para concepção de um modelo pedagógico final, com posterior experimentação final do mesmo para integração dos contributos. 


\section{Propostas do modelo pedagógico}

Esta parte do trabalho apresenta o modelo pedagógico construído a partir dos estudos preliminares (pesquisa exploratória e estudos de casos) em conjunto com a revisão bibliográfica realizada. Este foi formatado para fornecer algumas sugestões de estratégias a professores e pesquisadores interessados em utiliza-lo, orientando estes no desenvolvimento das atividades, com base na teoria da Sala de Aula Invertida e com o apoio de dispositivos móveis.

A evolução na construção do modelo pedagógico resultou em três propostas. O modelo pedagógico prévio ilustrado pela Figura 1, que foi construído a partir da revisão bibliográfica realizada, tendo como fundamentação a relação aluno, professor e sala de aula presente na teoria da Sala de Aula Invertida e posteriormente melhorado com os estudos preliminares baseando-se nos resultados das pesquisas exploratórias com estudos de casos, gerando um modelo pedagógico intermediário e por fim com os resultados das experimentações chegou-se ao modelo pedagógico final.

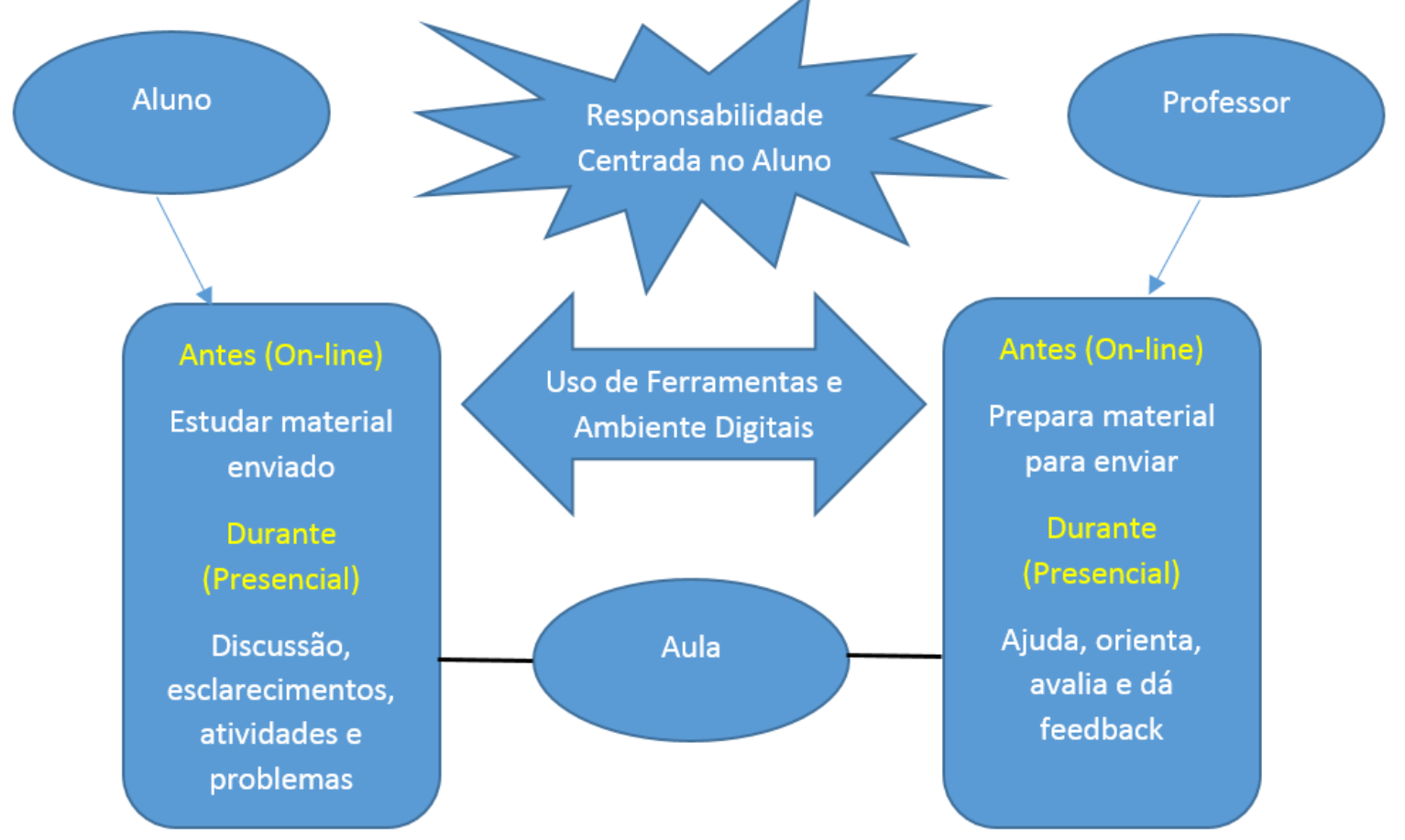

Figura 1: Modelo pedagógico prévio

Fonte: Elaborado pelos autores 
A Figura 1 apresenta a relação aluno, professor e aula, representando as atividades antes das aulas de forma on-line e durante as aulas de forma presencial, envolvendo as ferramentas e ambientes digitais, ampliando a responsabilidade centrada nos estudantes.

Os estudos de caso exploratórios preliminares, utilizaram os conceitos de Sala de Aula Invertida combinados com alguma tecnologia móvel, como Kahoot, WhatsApp e Facebook. Estes estudos de caráter exploratórios investigaram as possibilidades e potencialidades da utilização da Teoria da Sala de Aula Invertida com o auxílio de alguma Tecnologia Móvel.

Assim, como resultados encontrados nestes estudos preliminares e na revisão bibliográfica, tiveram destaque os seguintes elementos: As limitações e dificuldades de ordem financeira e técnica que podem excluir alguns alunos que não dispõem de smartphones, planos de internet em seus celulares ou mesmo internet em suas residências, dificultando o uso e principalmente o acesso dos alunos às ferramentas e aos recursos digitais disponíveis; Os benefícios significativos como o baixo custo, a acessibilidade, a interatividade e a aprendizagem colaborativa; A mediação pelo professor, como um fator fundamental, propondo temas e estimulando a participação dos alunos, identificando o contexto da sala de aula, dos alunos e da turma, estabelecendo regras e normas para utilização dos dispositivos móveis, deixando claro os objetivos e motivos das atividades propostas, verificando as limitações relevantes e os recursos tecnológicos necessários que serão utilizados, assim como, os papeis do professor e dos alunos neste processo; E por fim, a confirmação de que é fundamental um planejamento bem estruturado por parte do professor.

Desta forma, foram considerados estes resultados apresentados para a construção do modelo pedagógico intermediário, ilustrado na Figura 2. 


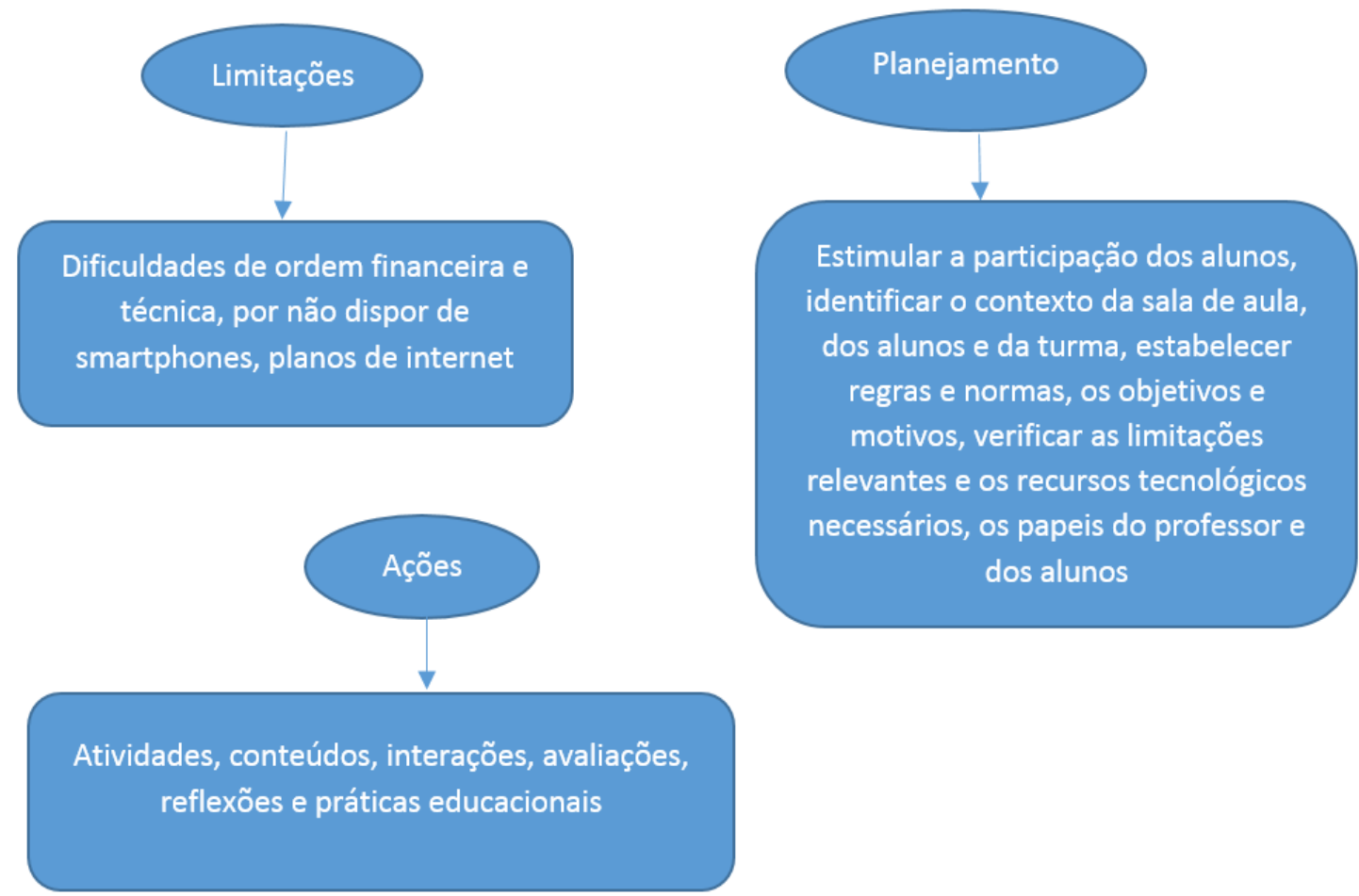

Figura 2: Modelo pedagógico intermediário

Fonte: Elaborado pelos autores

A Figura 2 apresenta agrupados os três níveis básicos de elementos encontrados no estudo, sendo eles: limitações, ações e planejamento. As limitações têm como objetivo encontrar as possíveis dificuldades, o planejamento foca em estruturar as atividades que serão desenvolvidas e as ações busca especificar como estas atividades serão aplicadas.

O modelo pedagógico final foi formatado levando-se em conta as experimentações realizadas nesta pesquisa. Neste presente estudo a AP foi reestruturada e fundamentada levando em consideração os conceitos da Sala de Aula Invertida, os aspectos relacionados a utilização dos dispositivos móveis, os estudos exploratórios preliminares realizados, e as experimentações realizadas incrementando e melhorando o modelo proposto. Ao final, a AP ficou estabelecida neste trabalho em seis aspectos, sendo eles: contexto, normatização, papeis, tecnologias, ações e limitações. Conforme visualiza-se na Figura 3. 
Res., Soc. Dev. 2019; 8(10):e318101384

ISSN 2525-3409 | DOI: http://dx.doi.org/10.33448/rsd-v8i10.1384
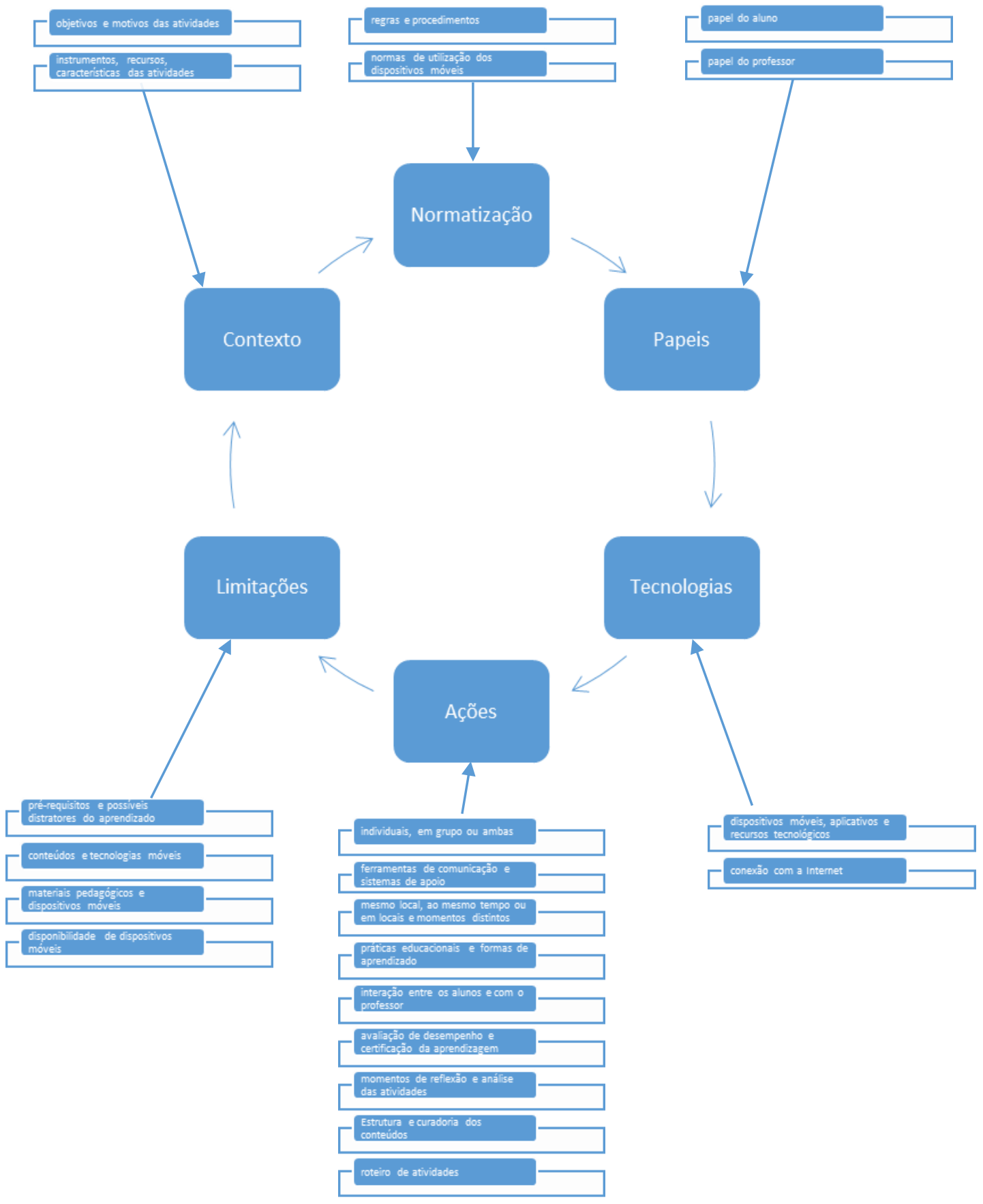

Figura 3: Modelo pedagógico final

Fonte: Elaborado pelos autores

A Figura 3 apresenta um desdobramento da Figura 2, onde buscou-se um maior detalhamento dos elementos envolvidos, ficando estes estabelecidos como: contexto, normatização, papeis, tecnologias, ações e limitações. Basicamente dividiu-se o planejamento em contexto, normatização, papeis, tecnologias, descrevendo características mais especificas 
envolvidas no modelo pedagógico.

A AP e as estratégias para a aplicação da AP foram definidas para as atividades com Sala de Aula Invertida e com apoio de dispositivos móveis. Observa-se que o modelo pedagógico proposto pode se adaptar e colaborar com o desenvolvimento de diversas atividades de m-learning envolvendo diferentes conteúdos e dispositivos móveis. Esclarece-se que são possíveis reestruturações e ajustamentos no modelo pedagógico proposto, mesmo durante a execução das ações planejadas, sempre levando em consideração as necessidades dos alunos, ressaltando ainda a importância do professor condutor neste processo.

\section{Experimentação do modelo pedagógico}

A Tabela 1 mostra os percentuais de respostas sobre se "A proposta apresentada envolvendo o uso de celulares e a metodologia da Sala de Aula Invertida, de maneira geral, foi importante para o aprendizado".

Tabela 1: A proposta foi importante para o aprendizado

\begin{tabular}{|c|c|c|c|c|c|}
\hline Curso & $\mathbf{5}$ & $\mathbf{4}$ & $\mathbf{3}$ & $\mathbf{2}$ & $\mathbf{1}$ \\
\hline SI & $43 \%$ & $47 \%$ & $10 \%$ & $0 \%$ & $0 \%$ \\
\hline
\end{tabular}

Fonte: Elaborado pelos autores

A Tabela 1 apresenta que cerca de 90\% dos alunos concordaram plenamente ou concordaram, mostrando uma forte aceitação por quase totalidade dos alunos, com poucos alunos que não concordam nem discordam e nenhum aluno contrário à proposta. Verificou-se que a maioria dos alunos concordaram que a atividade utilizando o modelo pedagógico proposto foi importante para o seu aprendizado.

Tabela 2: O modelo é adequado a seu objetivo

\begin{tabular}{cccccc|}
\hline \hline Curso & $\mathbf{5}$ & $\mathbf{4}$ & $\mathbf{3}$ & $\mathbf{2}$ & $\mathbf{1}$ \\
SI & $60 \%$ & $37 \%$ & $0 \%$ & $2 \%$ & $1 \%$ \\
\hline \hline
\end{tabular}

Fonte: Elaborado pelos autores 
Conforme ilustra a Tabela 2, observa-se que a maioria dos alunos afirmaram que o modelo está adequado a seu objetivo, que é contribuir com orientações para o planejamento e a realização de ações de m-learning por professores e pesquisadores de diferentes áreas.

Quando perguntados em uma questão aberta se consideravam que "O modelo pedagógico adotado pode colaborar para fins educativos, se sim qual? ”. A maioria dos alunos indicaram que o modelo pedagógico adotado colaborou com o aprendizado, intensificando algumas características positivas, tais como: autonomia, fixação dos conteúdos, rendimento dos alunos, participação, interesse pela matéria e interação entre os alunos. Apresentam-se, algumas das opiniões dos alunos sobre a implementação do modelo pedagógico proposto:

"As aulas estão bastante benéficas para o meu aprendizado";

“A nova metodologia aplicada em sala de aula nós ajuda muito, principalmente ao levarmos em conta que isso nos auxilia a sermos mais autônomos e capacitados";

"Facilitou o aprendizado instigando o aluno a participar mais da aula!";

"O uso do smartphone fez toda a diferença, contribuindo para o aprendizado, pois muitos estudam e trabalham, podendo acessar o conteúdo nos momentos de folga"

"O professor e as técnicas contribuíram para a aprendizagem";

"A metodologia é top, fiquei muito satisfeita".

Observou-se na maioria das respostas dos alunos que a utilização do modelo pedagógico proposto melhorou o rendimento da turma, promoveu maior autonomia e participação, os alunos demostraram uma excelente receptividade e aceitação em relação ao modelo e suas estratégias, e a atuação do professor foi bem recebida pelos alunos.

Quanto as limitações encontradas, observou-se que alguns alunos relataram à falta de tempo em acessar o conteúdo on-line, dificuldades com o material de estudo e a preferência pelas aulas tradicionais. Apresentam-se, algumas das justificativas dadas por alguns alunos:

"É uma metodologia interessante mas prefiro uma aula mais tradicional”;

"O conteúdo poderia ser apresentado de forma mais simples, e menos confuso";

"Boa metodologia, mas por trabalhar e estudar fica complicado acessar os conteúdos digitais".

\section{Considerações finais}

Este estudo apresenta a evolução na construção do modelo pedagógico ML-SAI, que resultou em três propostas consecutivas e sua experimentação. O modelo pedagógico teve três 
etapas de desenvolvimento, sendo eles o modelo pedagógico prévio, que foi construído a partir da revisão bibliográfica realizada, o modelo pedagógico intermediário que foi melhorado com os estudos preliminares, baseando-se nos resultados das pesquisas exploratórias de estudos de casos realizados com ferramentas digitais, e o modelo pedagógico final que foi aprimorado a medida que foram obtidos os resultados das experimentações realizadas em algumas disciplinas.

A proposição do ML-SAI é importante por levar em consideração os fundamentos da teoria da Sala de Aula Invertida, que vem apresentando ótimos resultados em trabalhos atuais, permitindo colaborar no planejamento e desenvolvimento consistente das práticas pedagógicas e melhor aproveitamento dos recursos tecnológicos. Assim, este modelo pedagógico foi formatado para fornecer algumas sugestões de estratégias a professores e pesquisadores interessados em utiliza-lo, orientando estes no desenvolvimento das atividades de m-learning. Sendo este bem aceito no teste realizado, com destaque para algumas características positivas intensificadas com a implementação do modelo pedagógico, tais como: autonomia, fixação dos conteúdos, rendimento dos alunos, participação, interesse pela matéria e interação entre os alunos.

Posteriormente, pretende-se realizar diversas novas experimentações, de modo a melhorar e consolidar o modelo pedagógico desenvolvido. O mesmo também será divulgado e disponibilizado em forma digital por meio de um aplicativo móvel, facilitando a sua ampla utilização por professores interessados.

\section{Referências}

Barcelos, R. J. S. Tarouco, L. \& Berch M. (2009). O uso de mobile learning no ensino de algoritmos. Revista Novas Tecnologias na Educação, Porto Alegre, v. 7, n. 2, p. 1-11.

Behar, P. A. (2009). Modelos pedagógicos em educação a distância. 311 p. Porto Alegre: Artmed.

Behar, P. A.; Passerino, L. \& Bernardi, M. (2007). Modelos Pedagógicos para Educação a Distância: pressupostos teóricos para a construção de objetos de aprendizagem. Revista Novas Tecnologias na Educação, Porto Alegre, v.5, n. 2, p. 1-12. 
Bergmann, J. \& Sams, A. A. (2016). Sala de aula invertida: uma metodologia ativa de aprendizagem. 1 ed. Rio de Janeiro: LTC.

Costa, E. Silva, A. P. Cordeiro, B. M. P. \& Silva, C. A. (2014). As tecnologias digitais chegaram! O que fazer? Formas inovadoras de aprender. In: L. G. Dantas, and M. J. Machado (Org.). Tecnologias e educação: perspectivas para a gestão, conhecimento e prática docente. 2 ed. São Paulo: FTD.

Gil, A. C. (1999). Métodos e técnicas de pesquisa social. São Paulo: Atlas.

Gil. A. C. (2002). Como elaborar projetos de pesquisa. 4. Ed. São Paulo: Atlas.

Moran, J. M. (2013). Novas tecnologias e mediação pedagógica. 21 ed. rev. E atual. Campinas, SP: Papirus.

Wains, S. I. \& Mahmood, W. (2008). Integrating m-learning with e-learning. 9th ACM SIGITE Conference on Information Technology Education, Cincinnati, USA, pp. 31-38.

\section{Porcentagem de contribuição de cada autor no manuscrito}

Ernane Rosa Martins - 50\%

Luís Borges Gouveia - 50\% 